\title{
Körperliche und psychische Folgen des Kokain- und Crackkonsums
}

\author{
Christian Haasen \\ Michael Prinzleve \\ Heike Zurhold \\ Rüdiger Schmolke \\ Julia Ilse
}

\author{
Somatic and Mental Health Consequences of Cocaine and Crack Use
}

\section{Zusammenfassung}

Die Zunahme des Kokain- und Crackkonsums erfordert eine differenzierte Auseinandersetzung mit den daraus resultierenden Begleiterscheinungen. In der Wirkung unterscheiden sich Kokain und Crack primär in der Geschwindigkeit des zentralnervösen Wirkungseintritts. Körperliche Komplikationen ergeben sich aus der vasokonstriktorischen Wirkung als auch durch das Abbauprodukt Kokaethylen bei gemeinsamem Konsum mit Alkohol. Da Kokain meistens nicht als Monosubstanz eingenommen wird, sind somatische Folgen selten monokausal auf das Kokain zurückzuführen. Das Missbrauchs- und Abhängigkeitspotenzial ist zum Teil auf die Wirkung auf spezifische Neurotransmittersysteme zurückzuführen. Das Craving und die Toleranzentwicklung sind bei Crackkonsum deutlicher ausgeprägt. Es besteht eine hohe Komorbidität vor allem mit Persönlichkeits- und affektiven Störungen. Bisher hat die medikamentöse Behandlung der Kokainabhängigkeit keine ausreichende Effektivität gezeigt, während verschiedene psychosoziale Interventionen eine positive Wirkung erzielten.

\section{Schliuisselwörter}

Kokain · Crack · Psychische Folgen · Somatische Komplikationen

\section{Abstract}

The increase in cocaine and crack use calls for a differentiated assessment of its health consequences. The primary difference in the effect of cocaine and crack lies in the speed of central nervous action. Somatic complications result due to vasoconstriction as well as related to the metabolite cocaethylene when consumed together with alcohol. Since cocaine is frequently not used alone, somatic complications seldom have a monocausal relationship to cocaine. The abuse and addictive potential is related to the action in specific neurotransmitter systems. Craving and development of tolerance are more frequent with crack use. There is a high rate of comorbidity especially with personality and affective disorders. Pharmacological treatment of cocaine addiction has not been shown to be sufficiently effective, while several psychosocial interventions have led to positive results.

\section{Key words}

Cocaine $\cdot$ Crack $\cdot$ Mental Health $\cdot$ Somatic Complications

\section{Einleitung}

Inwieweit sich der Konsum einer Substanz zu einem gesellschaftlichen „Problem“ entwickelt, hängt von einer Reihe von Faktoren und vor allem von deren Bewertung ab. Dazu zählen Faktoren wie allgemeine Prävalenz, Häufigkeit in der polizeilichen Statistik, gesundheitliche Folgen, aber auch die subjektive Bewertung der Schwierigkeiten im Umgang mit Abhängigen. Der Kokain- und Crackkonsum in Deutschland wird mittlerweile als ein ernsthaftes Problem angesehen [1]. Daher ist eine differenzierte Betrachtung des Kokain- und Crackkonsums notwendig, um eine erneute Diskussion anhand zum Teil widerlegter Mythen [2] vor allem um die Substanz Crack, wie sie in den USA in den 80er und 90er Jahren vorzufinden waren, zu vermeiden. Dazu soll der folgende Überblick über potenzielle Folgen des Kokain- und Crackkonsums anhand einer Auswertung v.a. der Literatur aus den letzten Jahren einen Beitrag leisten. 
Kokain ist das Alkaloid aus den Blättern der Kokapflanze (Erythroxylon Coca). Bei der Herstellung werden zunächst die getrockneten Kokablätter in einer Lösung von Kerosin, einer alkalischen Base (z.B. Kalziumoxid), Sodakarbonat und Schwefelsäure eingeweicht. Aus der daraus resultierenden Flüssigkeit entsteht dann die Kokapaste, eine braune Substanz. In der Kokapaste sind neben dem Kokain noch weitere Kokaalkaloide, aber auch Sulfate, Streckmittel und Verdünnungsmittel vorhanden. Durch eine Mischung mit Salzsäure kann das Alkaloid Kokain in der gängigsten Konsumform Kokainhydrochlorid, einem weißen Pulver, extrahiert werden. Im weiteren Text wird Kokain synonym für die Pulverform Kokainhydrochlorid benutzt.

Kokain wird klinisch-pharmakologisch zu der Gruppe der Stimulanzien gerechnet. Chemisch ist Kokain $\left(\mathrm{C}_{17} \mathrm{H}_{21} \mathrm{NO}_{4}\right)$ ein Benzoylmethylecgonin; Ecgonin ist verwandt mit dem Aminoalkohol des Atropins. Kokain hat die Grundstruktur eines synthetischen Lokalanästhetikums. Die Struktur beinhaltet ein hydrophiles tertiäres Amin und einen hydrophoben aromatischen Rest, getrennt durch eine intermediäre Alkylkette. Es wurde erstmals 1860 von Niemann chemisch isoliert und war das erste in der Medizin verwendete Lokalanästhetikum (produziert von der Firma Merck, Darmstadt).

\section{Was ist Crack?}

Crack kann entweder von Kokapaste (am ehesten in Lateinamerika) oder von Kokainhydrochlorid (in USA und Europa) gewonnen werden und ist somit nur eine Darreichungsform des Kokains und keine andere Substanz. Der Kokapaste müssen entsprechende Basen hinzugefügt werden, um Crack herzustellen. Das Kokainhydrochlorid wird mit Wasser und Bikarbonat oder Ammoniak gemischt und gekocht, bis eine braune oder beige Kristallform, Crack, ausfällt. Crack erhielt seinen Namen aufgrund des Geräusches, welches bei der Erhitzung zur Inhalation entsteht.

\section{Wirkung von Kokain bzw. Crack}

Die wichtigste Wirkung ist die Fähigkeit, den Beginn oder die Weiterleitung des nervlichen Impulses bei lokaler Applikation zu blockieren. Weiterhin kommt es zu einer Stimulation des zentralen Nervensystems. Das Missbrauchs- und Abhängigkeitspotenzial des Kokains ist wenigstens zum Teil auf die Wirkung auf spezifische Neurotransmittersysteme im Gehirn zurückzuführen. Die zentralen Neurotransmitter werden durch eine Inhibition der Wiederaufnahme von Norepinephrin, Serotonin als auch Dopamin beeinflusst. Außerdem wird die Freigabe von Norepinephrin und Dopamin erleichtert und die Tyrosinhydroxylase wird aktiviert. Die Nettowirkung entspricht einer Steigerung der Neurotransmitterspiegel. Die Aktivierung des dopaminergen und noradrenergen Stoffwechsels wird in Verbindung mit einer Stimmungsaufhellung gebracht, die Aktivierung des serotonergen Stoffwechsels mit Stimmung und Erregung. Dabei müssen beide Systeme für das Suchtpotenzial des Kokains verantwortlich sein, da Stoffe mit aktivierender Wirkung in nur einem der beiden Systeme kein Suchtpotenzial zeigen. Bei chro- nischem Kokainkonsum kommt es zu einer erhöhten Erregungsschwelle in dopaminergen Bereichen des Nucleus accumbens. Dies erklärt sich durch eine Downregulierung aufgrund des chronischen Konsums. Diese Dysregulation bewirkt eine Erschöpfung der dopaminergen Neurone, die auch in Verbindung mit der Anhedonie und Depression nach Kokainentzügen gebracht wird [3].

Der Wirkungsunterschied zwischen Crack und Kokainhydrochlorid ergibt sich aus der Einnahmeform: Kokain als Crack kann geraucht werden, welches mit einem schnelleren Wirkungseintritt einhergeht als bei dem nicht rauchbaren Kokainhydrochlorid, das entweder nasal oder intravenös konsumiert wird. Der von den Konsumenten erwünschte zentralnervöse Wirkungseintritt ist bei gerauchtem Kokain (Crack) schon nach 5 bis 10 Sekunden spürbar, bei intravenösem Konsum nach ca. 30 bis 120 Sekunden und bei nasalem Konsum nach 15 bis $60 \mathrm{Mi}-$ nuten [3]. Das schnellere Anfluten beim Rauchen von Crack erklärt sich über den kürzeren Weg von der Lunge bis zum Gehirn (direkt über das linke Herz), während der intravenöse Konsum zusätzlich über das rechte Herz und den Lungenkreislauf erfolgen muss. Die Inhalation eines „Steines“ von Crack entspricht der Einnahme von 50 bis 150 mg Kokain. Die Wirkung gipfelt nach ca. 5 bis 10 Minuten und fällt dann rapide ab, mit dem gefürchteten „Crash“ ca. 20 Minuten nach der Einnahme. Kokain wird im Wesentlichen in der Leber und im Plasma zu Benzoylecgonin metabolisiert, welches im Urin bis zu drei Tage, bei chronischem Gebrauch sogar bis zu drei Wochen nach dem letzten Konsum nachgewiesen werden kann.

\section{Körperliche Folgen}

Bei den körperlichen Folgen des Kokain- oder Crackkonsums muss unterschieden werden zwischen den Folgen aufgrund einer akuten (meist hohen) Einnahme im Sinne einer Intoxikation und den Folgen aufgrund einer chronischen Einnahme. Des Weiteren ist zu beachten, dass der Kokain- oder Crackkonsum nur in seltenen Fällen als Monokonsum verläuft. Daher gibt es eine Reihe von somatischen Komplikationen, die nicht direkt auf den Kokain- oder Crackkonsum zurückzuführen sind. Dabei stellt der Mischkonsum von Kokain mit Alkohol einen besonderen Fall dar: Bei diesem Mischkonsum entsteht im Körper die Substanz Kokaethylen als Abbauprodukt von Kokain. Kokaethylen ist eine psychoaktive Substanz mit einer deutlich höheren Toxizität als Kokain [4].

Aufgrund der hohen individuellen Varianz kann keine Dosisoder Blutspiegelmenge angegeben werden, die einer Toxizität gleichzusetzen wäre. Es wird von einer letalen Einzeldosis von 1,5 g ausgegangen [3]. Körperlich kann eine akute Kokainintoxikation schon bei einer Menge von $1 \mathrm{mg} / \mathrm{kg}$ Körpergewicht auftreten und zu einer Aktivierung des kardiovaskulären Systems mit Tachykardie, Hypertonie und Diaphorese führen. In schweren Fällen kann es zu einem Kreislaufkollaps kommen. Es werden ebenfalls Synkopen aufgrund einer Bradyarrhythmie beschrieben [5].

Des Weiteren werden eine Reihe von in der Literatur beschriebenen Komplikationen dargestellt, ohne dass dabei Aussagen über die Häufigkeit gemacht werden. Zum Teil sind diese Komplika- 
tionen nicht monokausal auf den Kokainkonsum zurückführbar, da sowohl andere exogene Faktoren als auch eine Prädisposition das Auftreten mit beeinflussen. Bei einem Kokainkonsum ist die Krampfschwelle reduziert mit der Gefahr eines epileptischen Krampfanfalles. Zusätzlich können zentralnervös Tremor, Hyperemesis und Hyperpyrexie auftreten, während das periphere Nervensystem mit Harn- und Stuhlretention, muskulären Verkrampfungen und Erröten reagieren kann. Es kann zu einer Ateminsuffizienz kommen, mit entsprechender Zyanose, bei schweren Fällen bis hin zum Atemstillstand.

Der Tod kardialer Zellen führt zu entsprechenden kardialen Beschwerden. Seltener kann es zu einer Rhabdomyolyse [6] mit akutem Nieren- und Leberversagen [7] kommen. Tödliche Verläufe sind auf eine respiratorische Insuffizienz mit Atemstillstand oder auf eine kardiovaskuläre Insuffizienz mit Herzstillstand zurückzuführen. Der zeitliche Verlauf bei einer Intoxikation zeigt meistens zuerst eine agitierte Unruhe mit Verwirrtheit, dann epileptische Anfälle und anschließend eine respiratorische oder kardiovaskuläre Insuffizienz, wobei die beiden ersten Schritte nicht notwendigerweise auftreten müssen.

Ein weiterer Bereich der somatischen Komplikationen ist auf die Vasokonstriktion, die durch das Kokain verursacht wird, zurückzuführen. Dabei kommt es häufig zu Vorstellungen in Notfallambulanzen aufgrund von Herzbeschwerden mit Brustschmerzen $[8,9]$. Hierbei werden Arrhythmien [10] und Hypertonus [11] häufiger beobachtet, seltener auch Herzinfarkt [12,13] oder zerebraler Insult. Bei der kardialen Ischämie wird neben der Vasokonstriktion auch eine Störung des biochemischen Stoffwechsels in den kardialen Zellen diskutiert [14]. Eine weitere bei Kokainkonsum berichtete Komplikation ist die akute ischämische Kolitis [15].

Ein besonderer Fall der Kokainintoxikation, jedoch nicht im Zusammenhang mit Crack, ist die im Magen-Darm-Trakt auftretende Ruptur der Plastikhülle, die für den Kokaintransport z. B. über Grenzen genutzt wird. Hierbei können bei Ruptur der Plastikhülle alle oben genannten Symptome auftreten, so dass eine intensiv-medizinische Überwachung erforderlich wird [16]. Eine besondere potenzielle Komplikation ist das Magenulkus, welches größere Dimensionen annehmen kann [17]. Bei diesen auch „body-packers“ genannten Menschen besteht bei Ruptur ein hohes Mortalitätsrisiko [3].

Bei chronischem Kokain- oder Crackkonsum können zusätzliche somatische Komplikationen hinzukommen. Da Kokain ein lokales Anästhetikum ist, werden Verletzungen z.B. im Rachenraum nicht wahrgenommen und können exazerbieren. Desgleichen wird eine toxische direkte Wirkung auf das Lungengewebe beschrieben, die jedoch im Vergleich zu der Inhalation von Freebase-Kokain aufgrund der Etherdämpfe wesentlich weniger toxisch ist. Bei chronischem Crack- oder Kokainkonsum kommen weiterhin Erscheinungen von Mangelernährung hinzu, wobei diese nur zum Teil auf die Unterdrückung des Hungergefühls durch Kokain zurückzuführen und eher Zeichen einer sozialen Marginalisierung sind. Ebenfalls nicht selten sind neurokognitive Defizite wie z.B. Konzentrationsstörungen, aber auch Schlafstörungen zu verzeichnen. Weiterhin wurden im Zusammenhang mit chronischem Kokainkonsum Fälle von Adenokarzi- nomen des Pankreas [18] und ischämischer Kolitis beschrieben [19].

Bisher sehr umstritten war die Frage, ob Kokain einerseits ein Risiko in der Schwangerschaft darstellt, andererseits ob es eine teratogene Wirkung auf den Fetus hat. Bezüglich der Schwangerschaft sind im Zusammenhang mit der Vasokonstriktion durch das Kokain folgende Komplikationen möglich [20]: ein reduzierter Blutfluss zwischen Uterus und Plazenta; akute und/oder chronische Plazentainsuffizienz; Hypertonus mit dem Risiko der Plazentaablösung; frühe Wehen mit der Gefahr einer Frühgeburt; höhere Rate an Spontanaborten. Da bei den KonsumentInnen jedoch Kokain oder Crack nur in seltenen Fällen die alleinige Substanz ist, wird ein kausaler Zusammenhang zwischen Kokainkonsum und Schwangerschaftskomplikationen, der nicht auch auf die anderen Substanzen zurückzuführen sein kann, infrage gestellt [21].

In Bezug auf die Teratogenität von Crack entstand in den USA Ende der 80er Jahre das „soziale Konstrukt“ [22] der Crack-Babys: Es wurde vermutet, dass der pränatale Kokainkonsum zu einer Behinderung des Kindes führt, welche sich durch langjährige Verhaltensauffälligkeiten und Lernbehinderung auszeichnen würde. Eine neue Metaanalyse [23] konnte jedoch zeigen, dass es trotz Risiken durch den pränatalen Kokainkonsum keinen Hinweis für eine direkte Teratogenität der Substanz Kokain bzw. Crack gibt. Die in vielen Studien beschriebenen Effekte seien indirekt über soziale und andere Gesundheitsfaktoren zu erklären.

Insgesamt sind die potenziellen körperlichen Folgen zwar ernst zu nehmende Komplikationen, die jedoch einerseits dosisabhängig sind und andererseits in den meisten Fällen im Zusammenhang mit parallel konsumierten psychotropen Substanzen stehen. Auch die Bedeutung der verwendeten Streckmittel ist im Zusammenhang mit den körperlichen Komplikationen nicht zu unterschätzen.

\section{Psychische Folgen}

Die meist von dem Konsumenten gewünschte Wirkung von Kokain oder Crack betrifft die zentralnervöse Wirkung. Es handelt sich dabei generell um angenehme Gefühle („high“) mit deutlicher Zunahme des Selbstwertgefühls, mit folgenden Komponenten: Euphorie, erhöhtes Energiegefühl, erhöhte Aufmerksamkeit, erhöhte sensorische Wahrnehmung (sexuell, hören, sehen, fühlen), reduzierter Appetit, reduziertes Schlafbedürfnis, Verschiebung von Müdigkeit, erhöhtes Selbstvertrauen. Dennoch können auch unerwünschte psychische Symptome auftreten, wie z.B. erhöhte Angst und Misstrauen, egozentrisches Verhalten, Dysphorie, anorektisches Verhalten und auch Wahnvorstellungen. Diese psychotischen Symptome erschweren wiederum die Diagnostik der zugrunde liegenden Suchtstörung [24].

Die Hauptdebatte in Bezug auf psychische Folgen bezieht sich auf die Abhängigkeit. Es gibt eine Minderheit von Konsumenten, die regelmäßig Kokain oder Crack konsumieren und bei denen ein süchtiges Verhaltensmuster deutlich wird. Inwieweit es beim Kokain oder Crack zu einer körperlichen Abhängigkeit kommt oder es sich eher um eine psychische Abhängigkeit handelt, ist bislang in der Fachliteratur umstritten. Diese Debatte be- 
ruht darauf, dass die bekannten vegetativen Entzugssymptome, die z.B. bei Alkohol- oder Heroinabhängigkeit bekannt sind, bei Kokainkonsumenten nicht beobachtet werden. Andererseits ist die Wirkung des Kokains in den verschiedenen Transmittersystemen des zentralen Nervensystems mittlerweile gut bekannt, so dass die Abhängigkeit über diesen Wirkungsmechanismus erklärt werden könnte.

Es gibt überzeugende Hinweise, dass das Phänomen Craving (starkes Verlangen, „Suchtdruck“), welches bei Kokainkonsumenten auftreten kann, eine neurobiologische Grundlage hat [3]: Die Erschöpfung der dopaminergen Neurone führt zu einer Anhedonie und depressiven Stimmungslage, mit einem darauf folgenden starken Verlangen nach der Substanz (Kokain), das zu einer erneuten Erregung des dopaminergen Systems führt. Somit entsteht ein Kreislauf, der für den Betroffenen in süchtiges Verhalten mündet. Dementsprechend zeigt sich ein linearer Zusammenhang zwischen negativem Affekt und Craving [25]. Dieses Craving ist aufgrund des schnelleren Wirkungseintritts und der kürzeren Wirkungszeit gerade bei Crackkonsum deutlicher ausgeprägt als bei den anderen Einnahmeformen des Kokainkonsums. Allerdings wird Craving auch bei Konsumenten beschrieben, die schon über mehrere Tage oder Wochen kein Kokain oder Crack konsumiert haben, wo sich das dopaminerge System eigentlich wieder normalisiert hat, so dass die neurobiologische Grundlage des Cravings nicht mehr existent sein dürfte.

Ein wichtiges Zeichen für das Bestehen einer Abhängigkeit ist das Kriterium der Toleranzentwicklung. Bei chronischem Kokainkonsum wird eine solche Toleranzentwicklung beschrieben, die beim Konsum in Form von Crack sehr schnell auftritt. Diese Toleranzentwicklung wird mit dem Craving in Verbindung gebracht, da das Verlangen nach der gleichen positiven Wirkung häufig schon bei der zweiten Crackpfeife aufgrund der Toleranzentwicklung nicht erfüllt werden kann, woraus die von Konsumenten häufig beschriebene Gier resultiert.

Das Craving führt gerade bei Crack zu einem speziellen Konsummuster: Es wird nahezu ununterbrochen geraucht, solange die finanziellen Möglichkeiten ausreichen. Eine solche intensive Konsumphase (auch „Binge“ genannt) kann wenige Stunden bis einige Tage andauern, in denen der Konsument ca. alle 15 bis 20 Minuten eine Pfeife raucht, alle anderen Tätigkeiten vernachlässigt und dabei weder schläft, isst oder mit anderen Menschen kommuniziert. Vor dem Hintergrund dieser Konsummuster entstanden die so genannten Crackhäuser - eher dunkle und ruhige Orte oder Wohnungen, in denen nur der Crackdealer sich von einem (meist in der Ecke sitzenden) Crackkonsumenten zum anderen bewegt. Der exzessiven Konsumphase folgt eine Phase der physischen Erschöpfung und Depressivität („Crash“), in der der Konsument je nach Länge des Binges bis zu 48 Stunden durchschläft. Die Zeitspanne zwischen solchen Binges kann zwischen wenigen Tagen bis Wochen andauern. In einer Studie konnte eine hohe Korrelation zwischen dem Ausmaß der Depressivität in der Crash-Phase und der Intensität des HighGefühls gezeigt werden [26].

Die Entzugserscheinungen nach Remission des Kokain- oder Crackkonsums beinhalten ein reduziertes Energieniveau, geringes Interesse an der Umwelt, depressive Stimmung mit oder ohne Angstsymptomen und eine verminderte Fähigkeit, Freude zu empfinden (Anhedonie). Diese Symptome sind zunächst nach Absetzen des Kokainkonsums eher mild, nehmen jedoch an Intensität in den darauf folgenden 12 bis 96 Stunden zu. Dieser Phase folgt ein starkes Schlafbedürfnis („Crash“). Anhedonie, Depression, Angst und Craving können über Tage bis Wochen bestehen bleiben. Vegetative Entzugserscheinungen, die eine begleitende Entzugsmedikation erforderlich machen, treten nicht auf.

Eine Wirkung, die vor allem Kokain in Form von Crack zugeschrieben wird, sind eine erhöhte Aggressivität und Gewalt. Hierbei wird vermutet, dass die durch Crack erzeugte Gier (Craving) illegale und gewalttätige Handlungen nach sich zieht mit dem Ziel, die finanziellen Mittel für die nächste Crackpfeife zu erwerben. Die Kriminalstatistik wird dabei als Beweis herangezogen: Der Anteil der Verbrechen im Zusammenhang mit Crack ist wesentlich höher als bei anderen Substanzen. Dennoch konnten Reinarman und Levine [27] zeigen, dass dieser höhere Anteil eher auf den Handel mit Crack zurückzuführen sei und weniger auf Handlungen durch die Konsumenten. Ebenfalls konnte gezeigt werden, dass eine erhöhte Aggressivität bei Crackkonsumenten nicht per se festzustellen ist, sondern vielmehr in Zusammenhang steht mit den soziokulturellen Faktoren des Milieus, in dem sich der Konsument aufhält.

Generell muss bei Kokainkonsumenten die hohe Komorbiditätsrate mit anderen psychiatrischen Störungen beachtet werden: Dies gilt in erster Linie in Bezug auf zusätzliche Suchtstörungen, wobei Alkohol mit einer Prävalenz von 60 bis $80 \%$ innerhalb der Gruppe von Kokainkonsumenten die Hauptsubstanz ausmacht. Dabei erfüllt der Alkoholkonsum bei Kokain- und Crackkonsumenten zum Teil die Funktion der Modulierung der Entzugserscheinungen [28], zum Teil dient initialer Alkoholkonsum als Trigger für den Kokain- und Crackkonsum. In einer Untersuchung in der offenen Drogenszene in Hamburg konnten Verthein et al. [29] clusteranalytisch zeigen, dass es zwei unterschiedliche Gruppen von Kokainkonsumenten waren: eine Gruppe mit zusätzlichem primären Heroinkonsum und eine Gruppe mit einem multiplen Substanzgebrauch mit hohem Alkoholanteil.

Ebenfalls sehr hoch ist die Komorbiditätsrate für Persönlichkeitsstörungen, vor allem Borderline-, narzisstische und dissoziale Persönlichkeitsstörungen [30,31]. Unterschätzt wird die Komorbidität mit posttraumatischen Belastungsstörungen $[32,33]$. Vor allem aber die Komorbidität mit einer depressiven Störung ist diagnostisch eine Herausforderung, da unter der Wirkung von Kokain die depressiven Symptome maskiert werden, während nach Abklingen der Kokainwirkung die depressiven Symptome sich nicht von der Crash-Phänomenologie unterscheiden lassen. Erst wenn eine depressive Symptomatik auch 4 bis 7 Tage nach Abklingen der Kokainwirkung andauert, kann von einer komorbiden depressiven Störung ausgegangen werden. In einem solchen Fall kann der Kokain- oder Crackkonsum im Sinne einer dysfunktionalen Selbstmedikation verstanden werden. Allerdings scheint auch hier der Alkoholkonsum eine Rolle zu spielen, da das Ausmaß der Depression bei Kokainkonsumenten eine hohe Korrelation mit dem Alkoholkonsum aufwies [34]. Dies zeigt den Forschungsbedarf in Bezug auf die Interaktion von affektiven und Suchtstörungen. Der chronische Kokain- oder Crackkonsum führt zu weiteren neuropsychologi- 
schen Defiziten, vor allem im Zusammenhang mit einem Alkoholkonsum [35], wie auch zu anderen psychischen Auffälligkeiten, wie z. B. Mutismus [36].

Es besteht vor allem bei Probierkonsumenten die Vorstellung, Kokain und Crack seien sexuelle Stimulanzien. In der akuten Situation bei nicht chronischen Konsumenten ist diese Vorstellung auch richtig: Die dopaminerge Stimulation führt zu einem erhöhten Energiegefühl und Libido. Bei chronischem Kokainkonsum jedoch trifft dieses nicht mehr zu: Es entsteht ein Verlust des sexuellen Interesses, eine eingeschränkte Orgasmusfähigkeit, die sich bei Kombination mit Alkohol noch weiter verschlechtert. In diesem Zusammenhang ist bei Crackkonsumenten die verringerte sexuelle Stimulation $\mathrm{zu}$ berücksichtigen: Aufgrund des erhöhten Cravings kommt es zu sozialem Desinteresse und einer obsessiven Beschäftigung nur mit dem Konsum.

Insgesamt fehlen, wie auch für die körperlichen Folgen, epidemiologische Aussagen über die Häufigkeit psychischer Folgen, die eine Risikoeinschätzung erlauben. Werden Repräsentativerhebungen aus den USA herangezogen, so ist das Abhängigkeitspotenzial für Kokain geringer als für Alkohol oder Heroin, während sich das für Crack vermutete höhere Abhängigkeitspotenzial am ehesten mit der sozialen Selektion der Konsumenten erklären lässt [27].

\section{Behandlung}

Die Kokainabhängigkeit bleibt derzeit eine der wenigen Abhängigkeitsstörungen, bei der die Psychopharmakologie bisher keine effektive Substanz hervorbringen konnte. Einige Studien [37] konnten eine Wirkung von Antidepressiva zeigen, v.a. trizyklischen Antidepressiva, aber auch selektiven Serotonin-Wiederaufnahmehemmern (SSRI), die jedoch wiederum in anderen Studien nicht repliziert werden konnten. Weiterhin gibt es Erfolgsmeldungen hinsichtlich des Einsatzes von Amantadine [38] und Methylphenidat [39,40]. Aufgrund der hohen Komorbiditätsrate können Effekte einer medikamentösen Behandlung auch in diesem Zusammenhang erklärt werden. Dennoch muss im individuellen Fall das Potenzial einer psychopharmakotherapeutischen Behandlung geprüft werden. Preuß et al. [41] liefern eine gute Übersicht der möglichen psychopharmakologischen Strategien bei Kokainabhängigkeit.

Unstrittig dagegen ist nach verschiedenen Studien die Wirkung der Psychotherapie: In einer sehr großen randomisierten Untersuchung konnte die Effektivität verschiedener Psychotherapierichtungen (kognitive Psychotherapie, psychodynamische Psychotherapie, Einzelberatung und gruppentherapeutische Beratung) für alle Behandlungsformen nachgewiesen werden [42] (siehe auch Beitrag von Mercer und Woody in diesem Heft). Auch fokussierte psychotherapeutische Interventionen, wie eine Aggressionsmanagementgruppe („anger management group“), haben sich als effektiv erwiesen [43]. Unzureichend entwickelt sind bisher niedrigschwellige Angebote, wie z.B. Ruheräume, Maßnahmen der Konsumkontrolle oder auch Mechanismen der Qualitätskontrolle der Substanzen [44].
Akupunktur (NADA-Protokoll: 3-5 Punkte am Ohr) hat sich als effektiv erwiesen, um einen therapeutischen Zugang zu den Kokain- bzw. Crackkonsumenten herzustellen und in der Folge eine weitere Abstinenzorientierung zu fördern [45-47]. Eine Substitutionsbehandlung ist bislang nicht ernsthaft in Erwägung gezogen worden, obwohl in Südamerika solche Ansätze mit Kokablättern bei Kokapastekonsumenten existieren [48].

Die Kokainabhängigkeit kann aufgrund der komplexen Zusammenhänge zwischen Wirkung der Substanz, Umweltfaktoren und persönlicher Konstellation des Betroffenen nicht auf eine therapeutische Maßnahme reduziert werden, sondern wird immer sowohl den individuellen als auch gesellschaftlichen Anforderungen gerecht werden müssen. Daher müssen in Zukunft bei der Entwicklung neuer therapeutischer Maßnahmen auch unkonventionelle Wege gegangen werden, um zu erfolgreichen Konzepten zu gelangen.

\section{Literatur}

${ }^{1}$ Stöver H. Bestandsaufnahme „Crack-Konsum“ in Deutschland: Verbreitung, Konsummuster, Risiken und Hilfeangebote. (www.archido. de). Bremen: BISDRO 2001

${ }^{2}$ Haasen C, Krausz M. Myths versus evidence with respect to cocaine and crack: Learning from the US experience. Eur Addict Res 2001; 7: $159-160$

${ }^{3}$ Platt JJ. Cocaine Addiction: Theory, Research, and Treatment. Cambridge MA: Harvard University Press 1997

${ }^{4}$ Karan LD, Haller DL, Schnoll SH. Cocaine and stimulants. In: Francis RJ, Miller SI (Hrsg). Clinical Textbook of Addictive Disorders. New York, London: The Guilford Press 1998; 168-201

${ }^{5}$ Castro VJ, Nacht R. Cocaine-induced bradyarrhythmia: an unsuspected cause of syncope. Chest 2000; 117: 275-277 (1)

${ }^{6}$ Ruttenber AJ, McAnally HB, Wetli CV. Cocaine-associated rhabdomyolysis and excited delirium: different stages of the same syndrome. Am J Forensic Med Pathol 1999; 20: 120-127 (2)

7 Volcy J, Nzerue CM, Oderinde A, Hewan-Iowe K. Cocaine-induced acute renal failure, hemolysis, and thrombocytopenia mimicking thrombotic thrombocytopenic purpura. Am J Kidney Dis 2000; 35: E3 (1)

${ }^{8}$ Hollander JE, Brooks DE, Valentine SM. Assessment of cocaine use in patients with chest pain syndromes. Arch Intern Med 1998; 158: $62-66(1)$

${ }^{9}$ Feldman JA, Bui LD, Mitchell PM, Perera TB, Lee VW, Bernard SA, Fish SS. The evaluation of cocaine-induced chest pain with acute myocardial perfusion imaging. Acad Emerg Med 1999; 6: 103 - 109 (2)

${ }^{10}$ Gamouras GA, Monir G, Plunkitt K, Gursoy S, Dreifus LS. Cocaine abuse: repolarization abnormalities and ventricular arrhythmias. Am J Med Sci 2000; 320: 9-12 (1)

${ }^{11}$ Mo W, Arruda JA, Dunea G, Singh AK. Cocaine-induced hypertension: role of the peripheral sympathetic system. Pharmacol Res 1999; 40: $139-145(2)$

12 Inyang VA, Cooper AJ, Hodgkinson DW. Cocaine induced myocardial infarction. J Accid Emerg Med 1999; 16: $374-375$ (5)

${ }^{13}$ Mittleman MA, Mintzer D, Maclure M, Tofler GH, Sherwood JB, Muller JE. Triggering of myocardial infarction by cocaine. Circulation 1999; 99: $2737-2741(21)$

${ }^{14}$ Devi BG, Chan AW. Effect of cocaine on cardiac biochemical functions. J Cardiovasc Pharmacol 1999; 33: 1 -6 (1)

${ }^{15}$ Papi C, Candia S, Masci P, Ciaco A, Montanti S, Capurso L. Acute ischaemic colitis following intravenous cocaine use. Ital J Gastroenterol Hepatol 1999; 31: $305-307$ (4)

${ }^{16}$ June R, Aks SE, Keys N, Wahl M. Medical outcome of cocaine bodystuffers. J Emerg Med 2000; 18: 221 - 224 (2)

17 Miller JS, Hendren SK, Liscum KR. Giant gastric ulcer in a body packer. J Trauma 1998; 45: 617-619 (3)

18 Duarte JG, do Nascimento AF, Pantoja JG, Chaves CP. Chronic inhaled cocaine abuse may predispose to the development of pancreatic adenocarcinoma. Am J Surg 1999; 178: 426 - 427 (5) 
${ }^{19}$ Linder JD, Monkemuller KE, Raijman I, Johnson L, Lazenby AJ, Wilcox CM. Cocaine-associated ischemic colitis. South Med J 2000; 93: 909-913 (9)

${ }^{20}$ Mooney EE, Boggess KA, Herbert WN, Layfield LJ. Placental pathology in patients using cocaine: an observational study. Obstet Gynecol 1998; 91: 925 - 929 (6)

${ }^{21}$ Cejtin HE, Young SA, Ungaretti J, Anciaux D, Imam S, Teopengco E, Sander CM. Effects of cocaine on the placenta. Pediatr Dev Pathol 1999; 2: $143-147(2)$

${ }^{22}$ Lyons P, Rittner B. The construction of the crack babies phenomenon as a social problem. Am J Orthopsychiatry 1998; 68: 313-320 (2)

${ }^{23}$ Frank DA, Augustyn M, Grant Knight W, Pell T, Zuckerman B. Growth, development, and behavior in early childhood following prenatal cocaine exposure: A systematic review. JAMA 2001; 285: 1613-1625

${ }^{24}$ Shaner A, Roberts LJ, Eckman TA, Racenstein JM, Tucker DE, Tsuang JW, Mintz J. Sources of diagnostic uncertainty for chronically psychotic cocaine abusers. Psychiatr Serv 1998; 49: 684-690 (5)

${ }^{25}$ Coffey SF, Dansky BS, Carrigan MH, Brady KT. Acute and protracted cocaine abstinence in an outpatient population: a prospective study of mood, sleep and withdrawal symptoms. Drug Alcohol Depend 2000; 59: $277-286(3)$

${ }^{26}$ Uslaner J, Kalechstein A, Richter T, Ling W, Newton T. Association of depressive symptoms during abstinence with the subjective high produced by cocaine. Am J Psychiatry 1999; 156: 1444-1446 (9)

${ }^{27}$ Reinarman C, Levine HG. Crack in America: Demon Drugs and Social Justice. Berkeley: University of California Press 1997

28 Magura S, Rosenblum A. Modulating effect of alcohol use on cocaine use. Addict Behav 2000; 25: 117 - 122 (1)

${ }^{29}$ Verthein U, Haasen C, Prinzleve M, Degkwitz P, Krausz M. Cocaine use and the utilisation of drug help services by consumers of the open drug scene in Hamburg. Eur Addict Res 2001; 7: 176-183

${ }^{30}$ McKay JR, Alterman AI, Cacciola JS, Mulvaney FD, O‘Brien CP. Prognostic significance of antisocial personality disorder in cocaine-dependent patients entering continuing care. J Nerv Ment Dis 2000; 188: 287-296 (5)

${ }^{31}$ Rutherford MJ, Cacciola JS, Alterman AI. Antisocial personality disorder and psychopathy in cocaine-dependent women. Am J Psychiatry 1999; 156: 849-856 (6)

32 Back S, Dansky BS, Coffey SF, Saladin ME, Sonne S, Brady KT. Cocaine dependence with and without post-traumatic stress disorder: a comparison of substance use, trauma history and psychiatric comorbidity. Am J Addict 2000; 9: 51 - 62 (1)

${ }^{33}$ Najavits LM, Gastfriend DR, Barber JP, Reif S, Muenz LR, Blaine J, Frank A, Crits-Christoph P, Thase M, Weiss RD. Cocaine dependence with and without PTSD among subjects in the National Institute on Drug Abuse Collaborative Cocaine Treatment Study. Am J Psychiatry 1998; 155: $214-219(2)$
${ }^{34}$ Brown RA, Monti PM, Myers MG, Martin RA, Rivinus T, Dubreuil ME, Rohsenow DJ. Depression among cocaine abusers in treatment: relation to cocaine and alcohol use and treatment outcome. Am J Psychiatry 1998; 155: 220-225 (2)

${ }^{35}$ Bolla KI, Funderburk FR, Cadet JL. Differential effects of cocaine and cocaine alcohol on neurocognitive performance. Neurology 2000; 54 : 2285-2292 (12)

${ }^{36}$ La-Monaca G, Donatelli A, Katz JL. A case of mutism subsequent to cocaine abuse. J Subst Abuse Treat 1999; 17: 109-112 (1-2)

${ }^{37}$ McDowell DM, Levin FR, Seracini AM, Nunes EV. Venlafaxine treatment of cocaine abusers with depressive disorders. Am J Drug Alcohol Abuse 2000; 26: 25 - 31 (1)

${ }^{38}$ Kampman KM, Volpicelli JR, Alterman AI, Cornish J, O‘Brien CP. Amantadine in the treatment of cocaine-dependent patients with severe withdrawal symptoms. Am J Psychiatry 2000; 157: 2052 - 2054 (12)

${ }^{39}$ Levin FR, Evans SM, McDowell DM, Kleber HD. Methylphenidate treatment for cocaine abusers with adult attention-deficit/hyperactivity disorder: a pilot study. J Clin Psychiatry 1998; 59: 300-305 (6)

${ }^{40}$ Wang GJ, Volkow ND, Hitzemann RJ, Wong C, Angrist B, Burr G, Pascani K, Pappas N, Lu A, Cooper T, Lieberman JA. Behavioral and cardiovascular effects of intravenous methylphenidate in normal subjects and cocaine abusers. Eur Addict Res 1997; 3: 49-54

${ }^{41}$ Preuss UW, Bahlmann M, Koller G, Soyka M. Die Behandlung der Kokainabhängigkeit: Intoxikation, Entzug und Rückfallprophylaxe. Fortschr Neurol Psychiatr 2000; 68: 224-238 (5)

42 Crits-Christoph P, Siqueland L, Blaine JD, Frank A, Luborsky L, Onken LS, Muenz L, Thase ME, Weiss RD, Gastfriend DR, Woody GE, Barber JP, Butler SF, Daley D, Salloum I, Bishop S, Najavits LM, Lis J. Psychosocial treatments for cocaine dependence: results of the National Institute on Drug Abuse Collaborative Cocaine Treatment Study. Arch Gen Psychiatry 1999; 56: 493-502

${ }^{43}$ Reilly PM, Shopshire MS. Anger management group treatment for cocaine dependence: preliminary outcomes. Am J Drug Alcohol Abuse 2000; 26: $161-177$ (2)

${ }^{44}$ Decorte T. Quality control by cocaine users: Underdeveloped harm reduction strategies. Eur Addict Res 2001; 7: 161 - 175

45 Avants SK, Margolin A, Holford TR, Kosten TR. A randomized controlled trial of auricular acupuncture for cocaine dependence. Arch Intern Med 2000; 160: 2305-2312 (15)

${ }^{46}$ Bullock ML, Kiresuk TJ, Pheley AM, Culliton PD, Lenz SK. Auricular acupuncture in the treatment of cocaine abuse. A study of efficacy and dosing. J Subst Abuse Treat 1999; 16: $31-38$ (1)

47 Otto KC, Quinn C, Sung YF. Auricular acupuncture as an adjunctive treatment for cocaine addiction. A pilot study. Am J Addict 1998; 7: $164-170(2)$

${ }^{48}$ Hurtado-Gumucio J. Coca leaf chewing as therapy for cocaine maintenance. Annales de Medecine Interne 2000; 151: BB44-48 (8) 